\title{
Comorbid substance abuse and brain morphology in recent-onset psychosis
}

Received: 3 January 2008 / Accepted: 26 May 2008/ Published online: 4 February 2009

Abstract The aim of the presented study was to compare schizophrenia and schizoaffective patients early in the course of the disease with and without comorbid substance abuse disorder (SUD vs. NSUD) with regard to brain morphology. In a prospective design 41 patients (20 SUD vs. 21 NSUD) diagnosed as recent-onset schizophrenia or schizoaffective disorder consecutively admitted to hospital received standardized psychopathological evaluation (BPRS, SANS, MADRS, CGI, GAF) and MRI scanning with volumetric measurement of superior temporal gyrus (STG), amygdala-hippocampal complex, and cingulum. Patients with SUD (primarily cannabis) were significantly younger, predominantly male and had a lower socioeconomic status. Despite less attentional impairment (SANS subscore) and elevated anxiety/ depression (BPRS subscore) in patients with SUD compared to NSUD, no other psychopathological differences could be detected. There were no differences in the assessed temporolimbic brain morphology between the two subgroups. In conclusion, in this study substance abuse in recent-onset psychosis had no effect on brain morphology and the earlier onset of

Dr. med. T. Wobrock, MD $(\varangle) \cdot$ P. Falkai, MD, PhD

Department of Psychiatry and Psychotherapy

Georg-August-University Göttingen

von-Siebold-Strasse 5

37075 Göttingen, Germany

Tel.: +49-551/39-9667

Fax: +49-551/39-3899

E-Mail: twobroc@gwdg.de

H. Sittinger, MD

Department of Psychiatry and Psychotherapy

SHG Sonnenbergkliniken

Sonnenbergstrasse

66121 Saarbrücken, Germany

B. Behrendt, $\mathrm{PhD} \cdot \mathrm{R}$. D'Amelio

$\vec{\infty}$ Department of Psychiatry and Psychotherapy

Z University Hospital of Saarland

Kirrberger Strasse

66421 Homburg, Saar, Germany psychosis in patients with comorbid SUD could not be explained by supposed accentuated brain abnormalities in temporolimbic regions.

Key words substance abuse c cannabis - recentonset schizophrenia - first-episode psychosis · brain morphology $\cdot$ magnetic resonance imaging

\section{Introduction}

Brain abnormalities identified in schizophrenia using neuropathological and neuroimaging techniques reveal evidence for structural and functional impairment in multiple brain regions focusing on frontal cortex, temporal cortex, thalamus, hippocampal complex, basal ganglia and even cerebellum [3]. Metaanalyses and reviews of published studies concerning volumetric measurements obtained by magnetic resonance imaging (MRI) e.g. [58, 71] have demonstrated ventricular enlargement and volume decrease especially of frontal and temporal lobe structures in schizophrenia, independent of the country of origin where the studies were conducted [14]. A metaanalysis of volumetric structural MRI studies investigating hippocampus reported a $4 \%$ bilateral volume reduction in schizophrenic patients [43], while some studies observed pronounced decrease of hippocampal structure only on the left side e.g., [57]. Abnormalities of temporal lobe structures linked to mesolimbic system were suggested as responsible for cognitive and emotional disturbance commonly seen in schizophrenia. Temporal volume reductions have been linked to clinical features [27]. Especially reduced bilateral hippocampal size has been associated with memory deficits, and the decrease of total volume of superior temporal gyrus (STG) correlates with the severity of thought disorder and auditory hallucinations $[4,57]$. In first-episode and neuroleptika-naive patients results of volumetric MRI studies 
are inconclusive [65]. Ventricular enlargement in neuroleptika-naive patients up to $20 \%$ compared to healthy controls was observed in most studies, but volume changes of cerebral structures are less conclusive. While most studies failed to detect a volume reduction in basal ganglia [32, 65], a decreased thalamus volume was frequently reported (in the range of $5-18 \%$ of thalamus volume compared to controls) [20, 23, 26]. Additionally gray matter of frontal and temporal lobe structures, especially entorhinal cortex and hippocampus, was found reduced $[27,30,63]$.

Substance abuse in individuals with schizophrenia is very common and has risen to the most prevalent comorbid psychiatric condition associated with schizophrenia [16]. Estimated life-time prevalence rates for substance abuse in schizophrenia range from approximately 10 to $65 \%$ [32, 41, 52]. Besides the legal substances tobacco and alcohol, cannabis seems to be the most illicit drug abused in schizophrenics and has been discussed as an important risk factor for developing schizophrenia [5, 72]. In recent epidemiological studies in European countries lifetime cannabis abuse in male schizophrenics under the age of 36 years was reported in up to 60\% [19]. Persisting comorbid substance use disorder (SUD) has been associated with a negative outcome like more frequent and longer periods of hospitalization, higher relapse rates even in first-episode patients, elevated EPS-rates, lower medication compliance, violence and unemployment $[18,33,35,41,45,51,57,60,62]$. Despite the high prevalence of comorbid SUD in schizophrenia and the proposed impact of cannabis abuse as an additional biological risk factor for the onset or development of schizophrenic psychosis, there is still few knowledge about the influence of substance abuse on brain morphology in schizophrenic patients.

\section{Subjects and methods}

In a prospective design we studied 68 inpatients with recent-onset psychosis consecutively admitted to hospital, in the years 19961999, fulfilling the following inclusion criteria: age between 18 and 40 years, first hospitalisation due to schizophrenic symptoms, illness duration since the onset of first psychotic symptoms not preceding more than 3 years, receiving antipsychotic medication not longer than 6 weeks prior to admission. All patients provided informed consent before entering the study and did not present a diagnosis of mental retardation. The study design was approved by the local ethics committee. Diagnoses were based on clinical interview according to ICD-10 criteria, and were consented within all members of the study group and the treating clinical psychiatrist, who was blind to the aims of the study. Patients were excluded from the study if they met any of the following criteria: organic central nervous system disorder (e.g., epilepsy, traumatic brain injury, infectious or toxic cerebrovascular disease), mental retardation, age less than 18 years or greater than 40 years, or inadequate knowledge of the German language. In addition to the clinical interview, drug screens (urine) were systematically performed on admission.
The final study sample comprised of 41 patients diagnosed as schizophrenia or schizoaffective disorder, who received the interview, the psychopathological rating and underwent MR imaging due to study protocol. The remaining 27 patients fulfilled exclusion criteria for MR imaging of refused participation in the MRI part of the study.

\section{Clinical ratings}

Recorded were socioepidemiological (age at recruitment, years of education, status of employment, family status, family history of psychiatric diseases) and clinical data. All patients received standardized psychopathological evaluation. For the assessment of positive, negative and general psychopathology the 18 -item version of the brief psychiatric rating scale (BPRS) [46], and the scale for the assessment of negative symptoms (SANS) [2] were used. For further analyses and statistical comparisons the five subscales anxiety/depression, anergia, thought disturbance, activation, and hostility/suspiciousness of the BPRS and the subscales of the SANS affective flattening, alogia, avolition/apathy, anhedonia and attentional impairment were used. Additionally, depressive symptoms were assessed by the montgomery asberg depression rating scale (MADRS) [40]. Global clinical evaluation was performed using the clinical global impressions scale (CGI) [28] for disease severity and the global assessment of functioning scale (GAF), used in DSM-IV (Axis V), measuring an individual's functional capacity [24]. Substance use patterns and severity of substance use was assessed by the German version of the European addiction severity index (EuropASI), a standardized clinical interview demonstrating high reliability and validity in patients with alcohol and drug dependence [53]. Premorbid intelligence was estimated by a test of verbal knowledge and language (MWT-B) [34].

\section{MRI acquisition and volumetric measurement}

Magnetic resonance imaging (MRI) was performed on a $1.5 \mathrm{~T}$ Scanner (Magneton Vision, Siemens) with coronal T1-weighted MPRAGE sequences (magnetisation prepared rapid gradient echo, repetition time $=15 \mathrm{~ms}$, echo time $=5 \mathrm{~ms}$, flip angle $=30^{\circ}, 1$ aquisition, matrix $160 \mathrm{~mm} \times 256 \mathrm{~mm}$ ) generating 220 consecutive slices of $1.0 \mathrm{~mm}$ thickness. Additionally T2-weighted DETSE sequences (double echo turbo spin echo) and T1-weighted spin echo sequences were acquired. The images were filtered for inter-slice intensity inhomogeneities. Brain images were realigned in three dimensions to standardize the differences in head tilt during image acquisition. Standardized scans were then reconstructed into entire contiguous coronal images with a 2.2-mm thickness, perpendicular to the anterior-posterior commissure (AC-PC) line. Manual area measurements were obtained using the Analyze 3.0 software tools. The regions of interest (ROIs) out of the temporolimbic structures were determined according to the literature e.g. [58]. Separate measurements were obtained for the left and right hemisphere. The person, who performed the manual measurement, was not involved in the clinical rating or treatment and blind to the patients diagnosis.

\section{Amygdala-hippocampal complex}

Neuroanatomical boundaries were based on operationalized criteria from postmortem histological work [7] and prior published studies e.g. [67]. The posterior boundary of the hippocampal formation began where an ovoid mass of gray matter appeared inferiomedially to the trigone of the lateral ventricle. The fasciola cinerea, gyrus fasciolaris, isthmus, and crus of the fornix were excluded from measurement. Following the interruption of the pulvinar by the crus of the fornix, all CA segments (CA1, CA2, CA3, CA4), dentate gyrus, alveus, parasubiculum, presubiculum, and 
subiculum proper were included in the measurements. The anterior boundary was the coronal slice posterior to the one where the cisterna pontis became clearly visible.

The amygdala was measured from the posterior slice in which it first became visible. Its anterior boundary was the slice in which the anterior commissure appeared.

\section{Superior temporal gyrus (STG)/heschl's gyrus (HG)}

The anterior border of the STG was defined by a vertical on the sagittal plane, where in coronal and axial view the STG could be clearly separated from the temporal pole. The posterior border was determined by a vertical line where the lateral fissure is capped by the supramarginal gyrus on the sagittal plane. The superior and inferior borders were determined by the CSF of the lateral fissure and the superior temporal sulcus, respectively. Tracing was performed on the coronal plane and controlled in axial view. The gyrus was bounded inferiorly by the superior temporal sulcus and superiorly by the CSF of the lateral fissure. The transversal gyrus (Heschl's Gyrus, HG) was included in the measurement, commencing from a point at the posterior margin of the insula next to the end of an opercular branch of the postcentral gyrus and terminating in the lateral border of STG.

\section{Cingulate gyrus}

As the posterior boundary of the cingulate gyrus a vertical line was chosen touching the most posterior tip of the outer surface of the callosal splenium. The anterior boundary was represented by a vertical line touching the most anterior tip of the outer surface of the callosal genu. The cingulate sulcus represented the superior boundary and the callosal sulcus was the inferior boundary of the cingulate gyrus. The cingulate was traced on alternating serial coronal slices. Sagittal and transaxial slices were also examined to obtain an overall view of the cingulate gyrus.

\section{Statistics}

Statistical analysis were carried out using the SPSS-PC package (Version 10.0) [44]. For the comparison of psychopathological items and MRI volumes (ROI) parametric (two-tailed $t$ test) and non-parametric procedures (Mann-Whitney- $U$ test) were used. The alpha level was set at 0.05 . In addition, an analysis of covariance (ANCOVA) was performed to test the influence of age, body height, gender, handedness and education on ROI volumes. For additional analysis the general linear model procedure (GLM) was calculated using a multivariate approach with ROI volumes as dependent variables, diagnostic subgroups (schizophrenic patients with and without comorbid SUD) as factors, while age and height served as covariates. Further interaction terms were included in the model as needed. The relations between regional volumes and subject characteristics (age, symptom severity) were examined by using Pearson correlation coefficients.

\section{Results}

According to structured clinical interview (EuropASI) lifetime drug abuse was reported by 32 of 68 patients with recent-onset schizophrenia or schizoaffective disorder. The abused drug (lifetime) was in 31 patients cannabis (THC), in nine patients stimulants (amphetamines, ecstasy), in six patient cocaine, in four patients opiates, in four patients hallucinogens, and in four patients alcohol. In all cases the patients experienced their first hospitalization due to schizo- phrenic psychopathology. In 20 patients delta-9tetrahydrocannabinol (THC) or metabolites, in two patients derivates of amphetamines, and in two patients both substances could be detected in urine at the time of admission.

The MRI study sample comprised of 41 patients with recent-onset psychosis (36 with schizophrenia and five with schizoaffective disorder). In this sample lifetime drug abuse was reported by 20 patients with recent-onset schizophrenia (THC 20 patients, amphetamines/ecstasy seven patients, opiates two patients, cocaine eight patients, hallucinogens one patient, alcohol two patients). About $90 \%$ of the patients reporting lifetime drug abuse consumed THC in the last 6 months before imaging, of those users $33 \%$ consumed THC at least weekly. $80 \%$ of the eight cocaine using patients and all amphetamine/ecstasy using patients reported abuse in the last 6 months before imaging. 16 patients with recent-onset schizophrenia and five patients with recent-onset schizoaffective disorder of the MRI study sample reported no lifetime drug abuse.

To rule out a selection bias we compared the 27 patients not undergoing MR imaging with the 41 patients of the MRI study sample with regard to sociodemographic and clinical characteristics. In the 27 patients the percentage of lifetime drug abusers (44.4\% vs. $49.0 \% ; P=$ NS., $\chi^{2}$ test) and schizoaffective patients $\left(14.8 \%\right.$ vs. $12.5 \% ; P=$ NS., $\chi^{2}$ test) were similar to that of the MRI study sample. In addition there were no differences in the substance abuse pattern (THC $>$ cocaine $>$ amphetamines/ecstasy), the age ( 25.2 vs. 26.95 years; $P=0.461)$, CGI (6.56 vs. $6.45 ; P=0.811)$, GAF $(28.37$ vs. $27.58 ; P=0.535)$, BPRS sum score (52.52 vs. $55.25 ; P=0.347)$, SANS composite score (39.07 vs. $42.79 ; P=0.518)$ or MADRS sum score ( 16.42 vs. $16.90 ; P=0.753$, all Mann-Whitney- $U$ test).

Sociodemographic characteristics of the MRI study simple divided by the subgroups of schizophrenic patients with comorbid substance use disorder (SUD) and without substance abuse (NSUD) are presented in Table 1. Compared with nonabusers the sample of substance abusers was significantly younger, predominantly male, and less married or living with a partner. Although the difference was not significant, schizophrenic patients with SUD had in most cases a lower level of education, had lower income and rate of employment, and revealed higher rates of first and second degree relatives with substance use disorders (mostly alcohol use disorder) and lower rates of family members with severe mental illness (SMI) (e.g. schizophrenic spectrum disorders, bipolar disorder and major depression).

Only slight differences in psychopathology concerning negative symptoms could be detected. Attentional impairment, expressed through the SANS subscore, was significantly less in abusers than in nonabusers on admission, while the SANS subscore 
Table 1 Comparison of sociodemographic characteristics

\begin{tabular}{|c|c|c|c|}
\hline & SUD & NSUD & $P$ \\
\hline No. of patients (\% of all patients) & $20(49 \%)$ & $21(51 \%)$ & - \\
\hline Age; in years (mean, SD) $)^{\mathrm{a}}$ & $23.7 \pm 6.0$ & $30.5 \pm 6.9$ & 0.002 \\
\hline Male gender; №. (\% of group) ${ }^{b}$ & $17(85 \%)$ & $10(48 \%)$ & 0.013 \\
\hline Unmarried (\% of group) ${ }^{\mathrm{b}}$ & $95 \%$ & $57 \%$ & 0.005 \\
\hline $\begin{array}{l}\text { Education less than } 10 \text { years } \\
\text { (\% of group })^{\mathrm{b}}\end{array}$ & $70 \%$ & $52 \%$ & 0.254 \\
\hline $\begin{array}{l}\text { Unemployed (\% of group) }{ }^{\mathrm{b}} \\
\text { Family history (in } \% \text { of group) }\end{array}$ & $35 \%$ & $14 \%$ & 0.127 \\
\hline Severe mental illness (SMI) & $5.0 \%$ & $19.1 \%$ & 0.175 \\
\hline Substance use disorder (SUD) & $20.0 \%$ & $4.8 \%$ & 0.070 \\
\hline Others & $5.0 \%$ & $14.3 \%$ & 0.323 \\
\hline
\end{tabular}

$P$ probability, SD standard deviation, SUD substance use disorder, NSUD no substance use disorder

a Mann-Whitney-U test

${ }^{b}$ Chi-square-test

Alogia and the SANS composite score were lower only at a trend level in schizophrenic patients with SUD compared to those without substance abuse. Schizophrenic patients with comorbid SUD showed more anxiety and depression assessed with the corresponding BPRS subscale compared to those without substance abuse. There were no other significant differences in positive, negative and general psychopathology assessed by BPRS total score and the remaining BPRS and SANS subscales. In addition, depressive symptoms assessed with the MADRS in schizophrenic patients with SUD were similar to those of patients without substance abuse. Clinical ratings of disease severity (CGI) and global functioning showed similar results in both patients groups. Table 2 presents more detailed results.

Table 2 Comparison of clinical characteristics on admission

\begin{tabular}{lccl}
\hline & SUD & NSUD & $P$ \\
\hline No of patients (\%) & $20(49 \%)$ & $21(51 \%)$ & - \\
CGI (mean, SD) & $6.4 \pm 0.8$ & $6.6 \pm 0.6$ & 0.318 \\
Premorbid IQ (language test) & $104 \pm 18$ & $102 \pm 10$ & 0.650 \\
$\quad$ (mean, SD) & $29.3 \pm 7.5$ & $26.1 \pm 6.9$ & 0.396 \\
GAF (mean, SD) & $56.3 \pm 10.7$ & $54.1 \pm 10.6$ & 0.557 \\
BPRS total score & & & \\
BPRS-subscales (mean, SD) & $12.9 \pm 3.7$ & $10.6 \pm 4.4$ & 0.029 \\
Anxiety/depression & $9.1 \pm 3.8$ & $10.5 \pm 4.9$ & 0.387 \\
Anergia & $14.6 \pm 3.7$ & $13.0 \pm 5.2$ & 0.266 \\
Thought disorder & $9.6 \pm 4.3$ & $10.1 \pm 4.8$ & 0.773 \\
Activation & $10.2 \pm 4.1$ & $9.8 \pm 3.4$ & 0.752 \\
Hostility/suspiciousness & $36.5 \pm 22.5$ & $48.3 \pm 22.8$ & 0.065 \\
SANS-composite score, (mean, SD) & & & \\
SANS-subscales (mean, SD) & $6.8 \pm 8.1$ & $9.4 \pm 8.7$ & 0.261 \\
Affective flattening & $5.0 \pm 3.7$ & $7.6 \pm 5.3$ & 0.057 \\
Alogia & $5.2 \pm 3.2$ & $4.9 \pm 3.1$ & 0.600 \\
Avolition/apathy & $8.2 \pm 4.9$ & $10.8 \pm 5.6$ & 0.130 \\
Anhedonia & $3.0 \pm 2.2$ & $4.8 \pm 2.6$ & 0.014 \\
Attentional impairment & $15.5 \pm 8.4$ & $18.8 \pm 8.8$ & 0.301 \\
MADRS (mean, SD) & & \\
\hline
\end{tabular}

$P$ probability, Mann-Whitney-U Test, SD standard deviation; SUD substance use disorder, NSUD no substance use disorder, CGI clinical global impressions, GAF global assessment of functioning, BPRS brief psychiatric rating scale, SANS scale for the assessment of negative symptoms, MADRS montgomery asberg depression rating scale
Since it has been demonstrated that chronic administration of antipsychotic medication could influence brain morphology (e.g. volume of caudate nucleus, 31) the pattern and dosage of administered psychotropic medication was compared between abusers (SUD) and nonabusers (NSUD) in our study sample. There was no significant differences between these subgroups in regard to this. Patients were treated mostly with first-generation antipsychotics alone or in combination with second-generation antipsychotics (SUD v. NSUD; $77 \%$ vs. $85 \%$ ). Scoring for extrapyramidal symptoms (EPS) revealed no significant differences between schizophrenic patients with and without SUD ( $P=\mathrm{NS}$, Mann-Whitney- $U$ test), additionally there was no significant difference in mean chlorpromazine equivalents $(P=\mathrm{NS}$, MannWhitney- $U$ test).

The measured regional volumes were normally distributed (Kolmogorov-Smirnov-Test). Comparing the volumes of the selected ROIs no significant differences could be detected between schizophrenic patients with and without comorbid SUD (two-tailed $t$ test, $P=\mathrm{NS}$ ). For additional analysis a general linear model procedure (GLM) was calculated using a multivariate approach with ROI volumes as dependent variables, diagnostic subgroups (schizophrenic patients with and without comorbid SUD) as factors, while age and body height served as covariates. Again, no significant influence of the subgroups on regional brain volumes could be found. Table 3 shows the ROI volumes (raw values in cubic centimetres) of both subgroups.

In a previous study we found in a sample of patients with recent-onset psychosis aged 18-59 years a decreased superior temporal gyrus asymmetry index in the subgroup of patients without SUD compared to those with comorbid SUD, suggesting more subtle brain abnormalities in psychotic patients without comorbid SUD [64]. Asymmetry index (AI) was calculated as followed: $\mathrm{AI}=\left(\right.$ Volume $_{\text {right }}-$ Volume $\left._{\text {left }}\right) /$ $0.5 \times\left(\right.$ Volume $_{\text {right }}+$ Volume $\left._{\text {left }}\right)$ [22]. In the here presented study there were no significant differences in regional asymmetry indices between the subgroups (Mann-Whitney- $U$ test, AHC-AI was not normally distributed) (for details see Table 4).

To detect an influence of brain morphology on psychopathology we performed correlations between the BPRS total score, SANS composite score, the related subscales and the volumes of the region of interests (Pearson's product-moment correlations). No significant association between psychopathological items and the volumes of the measured mesolimbic structures could be detected. In addition we performed correlations between a semiquantitative estimation of substance abuse (THC, cocaine, amphetamine/ecstasy, on a scale from 0 to 4 , whereas 0 means to have no problem and 4 to have severe problems resulting from substance abuse) and volumetric data. We find no significant correlation 
Table 3 Comparison of regional brain volumes (ROI)

\begin{tabular}{lccc}
\hline & \multicolumn{1}{l}{ SUD } & NSUD & $P$ \\
\hline No of patients (\%) & $20(49 \%)$ & $21(51 \%)$ & - \\
Left AHC (mean, SD), ccm & $3.27 \pm 0.58$ & $3.02 \pm 0.40$ & 0.134 \\
Right AHC (mean, SD), ccm & $3.28 \pm 0.61$ & $3.13 \pm 0.46$ & 0.380 \\
Left STG/HG (mean, SD), ccm & $17.72 \pm 2.32$ & $19.30 \pm 2.60$ & 0.121 \\
Right STG/HG (mean, SD), ccm & $19.52 \pm 2.28$ & $20.17 \pm 2.47$ & 0.820 \\
Left cingulate gyrus (mean, SD), ccm & $9.03 \pm 1.72$ & $8.86 \pm 1.74$ & 0.925 \\
Right cingulate gyrus (mean, SD), ccm & $8.61 \pm 1.47$ & $8.76 \pm 1.70$ & 0.799 \\
\hline
\end{tabular}

$P$ probability, two-tailed $t$ test, SD standard deviation, ccm cubic centimetre, SUD substance use disorder, NSUD no substance use disorder, $R O I$ region of interest, $A H C$ amygdala-hippocampal complex, STG/HG superior temporal gyrus/ heschl's gyrus
Table 4 Comparison of regional asymmetry indices (ROI)

\begin{tabular}{llll}
\hline & SUD & NSUD & \multicolumn{1}{c}{$P$} \\
\hline No of patients (\%) & $20(49 \%)$ & $21(51 \%)$ & - \\
Al-AHC (mean, SD) & $-0.035 \pm 0.199$ & $-0.0002 \pm 0.177$ & 0.130 \\
Al-STG/HG (mean, SD) & $+0.098 \pm 0.081$ & $+0.045 \pm 0.126$ & 0.157 \\
Al-cingulate gyrus (mean, SD) & $-0.043 \pm 0.190$ & $-0.011 \pm 0.167$ & 0.620
\end{tabular}

Asymmetry Index was calculated as followed:AI $=\left(\right.$ Volume $_{\text {right }}-$ Volume $\left._{\text {left }}\right) / 0.5 \times\left(\right.$ Volume $_{\text {right }}+$ Volume $\left._{\text {left }}\right)$ $P$ probability, Mann-Whitney- $U$ test, SD standard deviation, SUD substance use disorder, NSUD no substance use disorder, $A$ l asymmetry index, $R O I$ region of interest, $A H C$ amygdala-hippocampal complex, STG/HG superior temporal gyrus/heschl's gyrus between the severity of substance abuse and brain morphology.

As patients with schizoaffective disorder were only present in the nonabusing (NSUD) group, we compared these five patients with the remaining 16 schizophrenia NSUD patients with regard to psychopathology and MR volumetry. We found nearly identical mean scores for CGI, GAF, BPRS and MADRS in both subgroups. Patients with schizoaffective disorder presented less negative symptoms (SANS composite score), but the difference was not statistically significant (37.8 vs. $51.6 ; \quad P=0.354$; Mann-Whitney- $U$ test). The schizoaffective patients had greater ROI volumes than the schizophrenia patients, but this was statistically significant only for the left superior temporal gyrus $(21.1$ vs. $18.7 \mathrm{ccm}$; $P=0.042$, two-tailed $t$ test). This remained not significant after Bonferroni adjustment. If patients with schizoaffective disorder were excluded and only the schizophrenia patients in the NSUD $(n=16)$ and SUD $(n=20)$ group were compared, no significant volumetric differences between these subgroups could be detected.

\section{Discussion}

One of the aims of the study presented here was to investigate if there is a difference in subtle brain abnormalities between schizophrenia and schizoaffective patients with and without comorbid SUD even in the early course of the disorder. It could be expected that individuals with schizophrenia who use substances would show additional ventricular enlargement and reduced volume of mesolimbic structures due to literature describing poor outcome of schizophrenic patients with comorbid substance use disorder and in respect of the knowledge that the extended use of substances (e.g. alcohol, cocaine) can lead to cortical atrophy or reduction of gray matter concentration $[21,30]$. A previous study found significant volume reductions in schizophrenics with comorbid alcoholism compared to patients without this comorbidity [36].

However, our study could not confirm the suggestion of reduced regional brain volumes in schizophrenic patients with comorbid substance abuse. In our sample patients with recent-onset schizophrenia or schizoaffective disorder with comorbid substance abuse did not differ significantly in terms of volumetric morphometry in temporolimbic regions from schizophrenia patients without substance abuse. One explanation for the different findings could be that the preferred drug in our sample was cannabis and alcohol consumption was not extensive enough in the SUD patients to cause cortical atrophy or ventricular enlargement. The effect of cannabis consumption on brain volume is less clear as that of alcohol. The structural neuroimaging studies in cannabis abusers revealed conflicting results [48]. While two studies could not demonstrate an influence of cannabis on brain morphology, especially on hippocampus, compared to controls $[6,66]$, a third study found lower gray matter volumes in the right parahippocampal region, lower white matter density in the left parietal region as well as increased volumes of bilateral fusiform gyrus, right thalamus and left parahippocampal region in the group of chronic cannabis users [37]. 
Other investigators detected only less frontal whitematter volume percentage in the group of substance abusers (cannabis, opiates, cocaine) compared to healthy controls [54]. Another study without healthy control group revealed a significant correlation between age of first use and decreased total brain volume [68]. Subjects who started using marijuana before age 17, compared to those who started later, revealed smaller whole brain and percent cortical gray matter and larger percent white matter volumes [68].

A previous study examining brain volumes in cannabis-exposed patients with schizophrenia versus non-exposed patients with schizophrenia revealed no differences between the subgroups for total brain volume, total gray and white matter, ventricles, cerebellum and caudate except a decreased asymmetry of the lateral ventricles in the cannabis-exposed patients [10]. In a recent longitudinal study the same research group could demonstrate that first-episode schizophrenia patients with continued use of cannabis showed increased loss of cerebral gray matter volume and larger increases of in lateral and third ventricle volumes than healthy subjects and patients who did not use cannabis during the follow-up [50]. The amount of cannabis used were not specified in the publication while it was assessed based on patients and relatives estimation. No information on a doseresponse relationship was provided. Nevertheless, brain volumes of schizophrenia patients with and without cannabis abuse did not differ significantly at the beginning of the longitudinal study, although it has to be assumed that the patients abused cannabis several years before entering the study and the mean duration of schizophrenic illness is more than one year. This cross-sectional finding is in line with our results. However, a further study investigating the volumes of the superior frontal gyrus, anterior cingulated gyrus and orbital frontal lobe found less anterior cingulate gray matter in cannabis abusing first-episode schizophrenia patients compared with patients who did not abuse cannabis and healthy volunteers [64]. The association of reduced volume in anterior cingulate gyrus and the history of cannabis abuse in schizophrenia was more interpreted in the context of a disturbed function of the anterior cingulate with the consequence of poor decision-making and more compulsive drive towards drug use as a predisposition for substance abuse than as a toxic effect of cannabis itself. This contrasts with our results of no volumetric differences in the anterior cingulate between the two groups of schizophrenia patients. Although no data regarding the amount of cannabis consumption was given in both studies, one could speculate if differences in substance use are responsible for the conflicting results. Interestingly, neither the cross sectional study [64] nor the longitudinal study at the beginning [50] showed volumetric differences between first-episode schizophrenia patients and healthy controls except larger third ventricle in the schizophrenia group [50].

To our knowledge our study is the first comparing different temporolimbic brain volumes with the region of interest (ROI) method (anterior cingulate, STG, amygdala/hippocampus) in schizophrenia patients exposed to illegal drugs versus schizophrenia patients without a history of illegal drug abuse. The measured volumes of the selected ROIs (amygdalahippocampal complex, superior temporal gyrus, cingulum) are in the range of the presented data in literature $[38,39,43]$, taking into account that our measurement of STG includes the transversal gyrus, and the extraction of the cingulum was not restricted to the anterior part. Nevertheless the heterogeneity of the definition of the regional volume (ROIs) boundaries, differences in MRI techniques and volume extractions in previous studies make it difficult to compare our volumetric measurements. Decreases of gray and white matter volume over time may be related to the duration, type and cumulative dose of antipsychotic medication patients received [9]. Because the percentage of patients treated with second generation antipsychotics and the dose of conventional antipsychotics (chlorpromazine-equivalents) was similar in both subgroups, medication served not as biasing factor in our volumetric study. Long-term effects of antipsychotic medication or chronicity of illness are not to expect in a sample of patients with recent-onset schizophrenia.

The described sociodemographic characteristics in both patient subgroups are consistent with literature, revealing younger age, less education, less married and higher rates of unemployment in the substance abuser group [16, 17, 35, 41, 59]. The frequency of substance abuse in recent-onset schizophrenia observed in our study sample is also in line with the reported prevalence rates in first-episode schizophrenic spectrum disorder $[1,12,35,49]$. The pattern of abused drugs is similar to that observed in European studies, presenting high prevalence of cannabis and low prevalence of cocaine abuse in contrast to US surveys showing high rates of cocaine consumers [25, $42,56,57]$. Differences in psychopathology between abusers and nonabusers were reported inconsistently in the recent literature. Comorbid substance abuse has been associated with higher positive symptomatology $[8,13,45,61]$, lower negative symptoms [5, 47, 61], and fewer positive and negative symptoms [17]. Because the overall differences in psychopathology between substance using and nonusing schizophrenics were small [61], our results of similar scores in positive, negative and general symptoms are in line with literature.

Substance abuse may contribute to an earlier onset of schizophrenia as seen in our sample and as demonstrated in some [12, 41, 49], but not in the majority of previous studies [11, 17, 47, 62]. Substance abuse in first-episode schizophrenia 
(preferentially cannabis) may not lead to more brain abnormalities detectable by volumetric morphometry, which were frequently reported in schizophrenia. The results of the presented MRI study with no difference in brain morphology between substance abusing and nonabusing schizophrenia patient is in line with our previous findings of similar neurocognitive performance of these subgroups [70]. Preliminary analysis of own MRI data including first-episode patients with an age above 40 demonstrated even fewer subtle abnormalities of brain morphology in schizophrenia patients with comorbid substance abuse [69], suggesting that in this subgroup neurotransmitter changes or functional abnormalities due to substance abuse serve as a main risk factor for the onset of schizophrenia. Nevertheless, the now presented results support the hypothesis that the earlier psychosis onset of schizophrenia patients using illegal drugs does not depend on pronounced brain abnormalities.

There are several limitations of our study. The sample size may be too small to detect significant differences in volumetric measures. We did not match the group of abusers with nonabusers for age, gender or education and had no comparison with a healthy control group. The missing control group did not allow us to prove if the patients in our sample display volumetric reductions of brain volume at all compared to healthy subjects. Although we collected data about the pattern of substance abuse, we did not evaluate exactly the frequency and amount of substances consumed by the patients. In consequence we could not investigate the relationship of the quantity of abused drugs and changes in brain morphology in the way of a "dose-response" relationship.

Only the nonabusing group contained schizoaffective patients. This could have potentially biased our results. To minimize the bias we analysed the schizoaffective patients in the nonabusing group separately and found (nonsignificant) greater regional volumes in these patients. The inclusion of these patients leads to higher mean ROI volumes in the nonabusing group and would have made the initially hypothesized pronounced volumetric reduction in the abusing group even more detectable, if there would be such a difference between the groups.

We controlled the regional volumes for height (as covariates) in regard to the sufficient correlation of this parameter with the intracranial volume, but we did not measure the intracranial volume at all. Therefore we could not compare the relative volumes, which limits our study results. Unfortunately we calculated no inter-rater correlation, which is also a severe methodological limitation.

The intention of the study was comparative volumetric analysis of temporolimbic brain regions in a consecutive sample of patients with recent-onset schizophrenia eligible for inpatient treatment. Therefore our results should be interpreted as findings in a naturalistic and not experimental sample. Naturalistic designs may have more clinical implications than investigations of a strongly selected sample of patients. Nevertheless the presented results should be interpreted with caution, have to be replicated and need further investigation on larger samples including other neurobiological parameters (e.g. genetics, functional MRI, event related potentials) and longer follow-up periods to evaluate a potential neurodegenerative process in the course of schizophrenic disorder within the presented subgroups (SUD vs. NSUD).

In conclusion, early in the course of schizophrenia substance abuse (primarily cannabis) seems not to lead to more brain abnormalities and the earlier onset of psychosis in patients with comorbid substance use compared to patients without substance abuse could not be explained by the initially supposed reduced brain volumes in temporolimbic regions reflecting potentially higher disturbances in neurodevelopment.

\section{References}

1. Addington J, Addington D (1998) Effect of substance misuse in early psychosis. Br J Psychiatry 172(Suppl. 33):134-136

2. Andreasen NC (1989) Scale for the assessment of negative symptoms (SANS). Br J Psychiatry 155(Suppl 7):53-58

3. Andreasen NC (2000) Schizophrenia: the fundamental questions. Brain Res Rev 31:106-112

4. Barta PE, Pearlson GD, Powers RE, Richards SS, Tune LE (1990) Auditory hallucinations and smaller superior temporal gyral volume in schizophrenia. Am J Psychiatry 147:1457-1462

5. Bersani G, Orlandi V, Kotzalidis GD, Pancheri P (2002) Cannabis and schizophrenia: impact on onset, course, psychopathology and outcomes. Eur Arch Psychiatry Clin Neurosci 252:86-92

6. Block RI, O'Leary DS, Ehrhardt JC, Augustinack JC, Ghoneim MM, Arndt S, Hall JA (2000) Effects of frequent marijuana use on brain tissue volumes and composition. Neuroreport 11:491596

7. Bogerts B, Falkai P, Haupts M, Greve B, Ernst S, TapernonFranz U, Heinzmann U (1990) Post-mortem volume measurements of limbic system and basal ganglia structures in chronic schizophrenics. Initial results from a new brain collection. Schizophr Res 3:295-301

8. Bühler B, Hambrecht M, Löffler W, van der Heiden W, Häfner $\mathrm{H}$ (2002) Precipitation and determination of the onset and course of schizophrenia by substance abuse-a retrospective and prospective study of 232 population-based first illness episodes. Schizophr Res 54(3):243-251

9. Cahn W, Hulshoff Pol HE, Lems EBT, van Haren NEM, Schnack HG, van der Linden JA, Schothorst PF, van Engeland H, Kahn RS (2002) Brain volume changes in first-episode schizophrenia. A 1-year follow-up study. Arch Gen Psychiatry 59:1002-1010

10. Cahn W, Hulshoff Pol HE, Caspers E, van Haren NE, Schnack HG, Kahn RS (2004) Cannabis and brain morphology in recentonset schizophrenia. Schizophr Res 67(2/3):305-307

11. Cantor-Graae E, Nordstrom LG, McNeil TF (2001) Substance abuse in schizophrenia: a review of the literature and a study of correlates in Sweden. Schizophr Res 48:69-82

12. Cantwell R, Brewin J, Glazebrook C, Dalkin T, Fox R, Medley I, Harrison G (1999) Prevalence of substance misuse in firstepisode psychosis. Br J Psychiatry 174:150-153

13. Caspari D (1999) Cannabis and schizophrenia: results of a follow-up study. Eur Arch Psychiatry Clin Neurosci 249:45-49 
14. Chua SE, Lam IW, Tai KS, Cheung C, Tang WN, Chen EY, Lee PW, Chan FL, Lieh-Mak F, McKenna PJ (2003) Brain morphological abnormality in schizophrenia is independent of country of origin. Acta Psychiatr Scand 108(4):269-275

15. Cleghorn JM, Kaplan RD, Szechtman B, Szechtman H, Brown GM, Franco S (1991) Substance abuse and schizophrenia: effects on symptoms but not on neurocognitive function. J Clin Psychiatry 52:26-30

16. Cuffel BJ, Heithoff KA, Lawson W (1993) Correlates of patterns of substance abuse among patients with schizophrenia. Hosp Com Psychiatry 44:247-251

17. Dixon L, Haas G, Weiden PJ, Sweeney J, Frances AJ (1991) Drug abuse in schizophrenic patients: clinical correlates and reasons for use. Am J Psychiatry 14:224-230

18. Drake RE, Mueser KT (2000) Psychosocial approaches to dual diagnosis. Schizophr Bull 26:105-118

19. Duke PJ, Pantelis C, McPhillips MA, Barnes TR (2001) Comorbid non-alcohol substance misuse among people with schizophrenia: epidemiological study in central London. Br J Psychiatry 179:509-513

20. Ettinger U, Chitnis XA, Kumari V, Fannon DG, Sumich AL, O'Ceallaigh S, Doku VC, Sharma T (2001) Magnetic resonance imaging of the thalamus in first-episode psychosis. Am J Psychiatry 158(1):116-118

21. Franklin TR, Acton PD, Maldjian JA, Gray JD, Croft JR, Dackis CA, O'Brien CP, Childress AR (2002) Decreased gray matter concentration in the insular, orbitofrontal, cingulate, and temporal cortices of cocaine patients. Biol Psychiatry 51(2):134-142

22. Galaburda AM, Corsiglia J, Rosen GD, Sherman GF (1987) Planum temporale asymmetry: reappraisal since Geschwind and Levitsky. Neuropsychologia 25:853-868

23. Gilbert AR, Rosenberg DR, Harenski K, Spencer S, Sweeney JA, Keshavan MS (2001) Thalamic volumes in patients with firstepisode schizophrenia. Am J Psychiatry 158(4):618-624

24. Goldman HH, Skodal AE, Lave TR (1992) Revising axis V for DSM-IV: a review of measures of social functioning. Am J Psychiatry 149:1148-1156

25. Graham HL, Maslin J, Copello A, Birchwood M, Mueser K, McGovern D, Georgiou G (2001) Drug and alcohol problems amongst individuals with severe mental health problems in an inner city area of the UK. Soc Psychiatry Psychiatr Epidemiol 36(9):448-455

26. Gur RE, Cowell P, Turetsky BI, Gallacher F, Cannon T, Bilker W, Gur RC (1998) A follow-up magnetic resonance imaging study of schizophrenia. Relationship of neuroanatomical changes to clinical and neurobehavioral measures. Arch Gen Psychiatry 55(2):145-152

27. Gur RE, Turetsky BI, Cowell PE, Finkelman C, Maany V, Grossman RI, Arnold SE, Bilker WB, Gur RC (2000) Temporolimbic volume reductions in schizophrenia. Arch Gen Psychiatry 57(8):769-775

28. Guy W, Bonato RR (eds) (1976) CGI. Clinical global impressions. Manual for the EDCEU assessment battery, Rev edn. Chevy Chase, Maryland, pp 12-1-12-6

29. Jernigan TL, Butters N, DiTraglia G, Schafer K, Smith T, Irwin M, Grant I, Schuckit M, Cermak LS (1991) Reduced cerebral grey matter observed in alcoholics using magnetic resonance imaging. Alcohol Clin Exp Res 15(3):418-427

30. Joyal CC, Laakso MP, Tiihonen J, Syvalahti E, Vilkman H, Laakso A, Alakare B, Rakkolainen V, Salokangas RK, Hietala J (2002) A volumetric MRI study of the entorhinal cortex in first episode neuroleptic-naive schizophrenia. Biol Psychiatry 51(12):1005-1007

31. Keshavan MS, Rosenberg D, Sweeney JA, Pettegrew JW (1998) Decreased caudate volume in neuroleptic-naïve psychotic patients. Am J Psychiatry 155:774-778

32. Kovasznay B, Fleischer J, Tanenberg-Karant M, Jandorf L, Miller AD, Bromet E (1997) Substance use disorder and the early course of illness in schizophrenia and affective psychosis. Schizophr Bull 23:195-201

33. Lacro JP, Dunn LB, Dolder CR, Leckband SG, Jeste DV (2002) Prevalence of and risk factors for medication nonadherence in patients with schizophrenia: a comprehensive review of recent literature. J Clin Psychiatry 63:892-909
34. Lehrl S (1977) Mehrfachwahl-Wortschatz-Intelligenztest. B-version. Erlangen, Straube

35. Linszen DH, Dingemans PM, Lenior ME (1994) Cannabis abuse and course of recent-onset schizophrenic disorders. Arch Gen Psychiatry 51:273-279

36. Mathalon DH, Pfefferbaum A, Lim KO, Rosenbloom MJ, Sullivan EV (2003) Compounded brain volume deficits in schizophrenia-alcoholism comorbidity. Arch Gen Psychiatry 60(3):245-252

37. Matochik JA, Eldreth DA, Cadet JL, Bolla KI (2005) Altered brain tissue composition in heavy marijuana users. Drug Alcohol Depend 77:23-30

38. Mitelman SA, Shihabuddin L, Brickman AM, Hazlett EA, Buchsbaum MS (2005) Volume of the cingulate and outcome in schizophrenia. Schizophr Res 72(2/3):91-108

39. Molina V, Sanz J, Sarramea F, Luque R, Benito C, Palomo T (2006) Dorsolateral prefrontal and superior temporal volume deficits in first-episode psychoses that evolve into schizophrenia. Eur Arch Psychiatry Clin Neurosci 256(2):106-111

40. Montgomery SA, Asberg M (1979) A new depression scale designed to be sensitive to change. Br J Psychiatry 134: 382-389

41. Mueser KT, Yarnold PR, Levinson DR, Singh H, Bellack AS, Kee K, Morrison RL, Yadalam DG (1990) Prevalence of substance abuse in schizophrenia: demographic and clinical correlates. Schizophr Bull 16:31-56

42. Mueser KT, Drake RE, Wallach MA (1998) Dual diagnosis: a review of etiological theories. Addict Behav 23:717-734

43. Nelson MD, Saykin AJ, Flashman LA, Riordan HJ (1998) Hippocampal volume reduction in schizophrenia as assessed by magnetic resonance imaging: a meta-analytic study. Arch Gen Psychiatry 55(5):433-440

44. Norusis MJ (2000) SPSS 10.0 guide to data analysis. PrenticeHall, New Jersey

45. Olivera AA, Kiefer MW, Manley NK (1990) Tardive dyskinesia in psychiatric patients with substance abuse. Am J Alcohol Abuse 16:57-66

46. Overall JD, Gorham DR (1962) The brief psychiatric rating scale. Psychol Rep 10:799-812

47. Peralta V, Cuesta MJ (1992) Influence of cannabis abuse on schizophrenic psychopathology. Acta Psychiatr Scand 85:127130

48. Quickfall J, Crockford D (2006) Brain neuroimaging in cannabis use: a review. J Neuropsychiatry Clin Neurosci 18:318-332

49. Rabinowitz J, Bromet EJ, Lavelle J, Carlson G, Kovasznay B, Schwartz JE (1998) Prevalence and severity of substance use disorders and onset of psychosis in first-admission psychotic patients. Psychol Med 28(6):1411-1419

50. Rais M, Cahn W, Van Haren N, Schnack H, Caspers E, Hulshoff Pol H, Kahn R (2008) Excessive brain volume loss over time in cannabis-using first-episode schizophrenia patients. Am J Psychiatry 165:490-496

51. Rasanen P, Tiihonen J, Isohanni M, Rantakallio P, Lehtonen J, Moring J (1998) Schizophrenia, alcohol abuse, and violent behavior: a 26-year followup study of an unselected birth cohort. Schizophr Bull 24(3):437-441

52. Regier DA, Farmer ME, Rae DS, Locke BZ, Keith SJ, Judd LL, Goodwin FK (1990) Comorbidity of mental disorders with alcohol and other drug abuse: results form the epidemiologic catchment area (ECA) study. JAMA 264:2511-2518

53. Scheurich A, Müller MJ, Wetzel H Scheurich A, Muller MJ, Wetzel $\mathrm{H}$, Anghelescu I, Klawe C, Ruppe A, Lorch B, Himmerich H, Heidenreich M, Schmid G, Hautzinger M, Szegedi A (2000) Reliability and validity of the German version of the European addiction severity index (EuropASI). J Stud Alcohol 61:916-919

54. Schlaepfer TE, Lancaster E, Heidbreder R, Strain EC, Kosel M, Fisch HU, Pearlson GD (2006) Decreased frontal white-matter volume in chronic substance abuse. Int J Neuropsychopharmacol 9(2):147-153

55. Serper MR, Alpert M, Richardson WA, Dickson S, Allen MH, Werner A (1995) Clinical effects of recent cocaine use on patients with acute schizophrenia. Am J Psychiatry 152:1464-1469 
56. Sevy S, Kay SR, Opler LA, van Praag HM (1990) Significance of cocaine history in schizophrenia. J Nerv Ment Dis 178:642-648

57. Shenton ME, Kikinis R, Jolesz FA, Pollak SD, LeMay M, Wible CG, Hokama H, Martin J, Metcalf D, Coleman M, McCarley RW (1992) Abnormalities of the left temporal lobe and thought disorder in schizophrenia: a quantitative magnetic resonance imaging study. N Engl J Med 327: 604-612

58. Shenton ME, Dickey CC, Frumin M, McCarley RW (2001) A review of MRI findings in schizophrenia. Schizophr Res 49:1-52

59. Soyka M, Albus M, Kathmann N, Finelli A, Hofstetter S, Holzbach R, Immler B, Sand P (1993) Prevalence of alcohol and drug abuse in schizophrenia inpatients. Eur Arch Psychiatry Clin Neurosci 242: 272-362

60. Soyka M (2000) Substance misuse, psychiatric disorder and violent and disturbed behaviour. Br J Psychiatry 176:345-350

61. Soyka M, Albus M, Immler B, Kathmann N, Hippius H (2001) Psychopathology in dual diagnosis and non-addicted schizophrenics-are there differences? Eur Arch Psychiatry Clin Neurosci 251:232-238

62. Swofford CD, Kasckow JW, Scheller-Gilkey G, Inderbitzin LB (1996) Substance use: a powerful predictor of relapse in schizophrenia. Schizophr Res 20(1/2):145-151

63. Szeszko PR, Goldberg E, Gunduz-Bruce H, Ashtari M, Robinson D, Malhotra AK, Lencz T, Bates J, Crandall DT, Kane JM, Bilder RM (2003) Smaller anterior hippocampal formation volume in antipsychotic-naive patients with first-episode schizophrenia. Am J Psychiatry 160(12):2190-2197

64. Szeszko PR, Robinson D, Sevy S, Kumra S, Rupp CI, Betensky JD, Lencz T, Ashtari M, Kane JM, Malhotra AK, Gunduz-Bruce H, Napolitano B, Bilder RM (2007) Anterior cingulate greymatter deficits and cannabis use in first-episode schizophrenia. Br J Psychiatry 190:230-236
65. Torrey EF (2002) Studies of individuals with schizophrenia never treated with antipsychotic medications: a review. Schizophr Res 58:101-115

66. Tzilos GK, Cintron CB, Wood JB, Simpson NS, Young AD, Pope HG Jr, Yurgelun-Todd DA (2005) Lack of hippocampal volume change in long-term heavy cannabis users. Am J Addict $14(1): 64-72$

67. Watson C, Andermann F, Gloor P, Jones-Gotman M, Peters T, Evans A, Olivier A, Melanson D, Leroux G (1992) Anatomic basis of amygdaloid and hippocampal volume measurement by magnetic resonance imaging. Neurology 42:1743-1750

68. Wilson W, Mathew R, Turkington T, Hawk T, Coleman RE, Provenzale J (2000) Brain morphological changes and early marijuana use: a magnetic resonance and positron emission tomography study. J Addict Dis 19(1):1-22

69. Wobrock T, Sittinger H, Falkai P, Behrendt B, Caspari D (2004) Comorbid substance abuse in recent-onset schizophrenia: brain morphology and neuropsychological evaluation. Schizophr Res 67/1S:221

70. Wobrock T, Sittinger H, Behrendt B, D'Amelio R, Falkai P, Caspari D (2007) Comorbid substance abuse and neurocognitive function in recent-onset schizophrenia. Eur Arch Psychiatry Clin Neurosci 257(4):203-210

71. Wright IC, Rabe-Hesketh S, Woodruff PWR, David AS, Murray RM, Bullmore ET (2000) Meta-analysis of regional brain volumes in schizophrenia. Am J Psychiatry 157:16-25

72. Zammit S, Allebeck P, Andreasson S, Lundberg I, Lewis G (2002) Self reported cannabis use as a risk factor for schizophrenia in Swedish conscripts of 1969: historical cohort study. BMJ 325: 1199 